\title{
Can a quantum field theory ontology help resolve the problem of consciousness?
}

\author{
Anand Rangarajan \\ Dept. of Computer and Information Science and Engineering \\ University of Florida
}

\begin{abstract}
The hard problem of consciousness arises in most incarnations of present day physicalism. Why should certain physical processes necessarily be accompanied by experience? One possible response is that physicalism itself should be modified in order to accommodate experience: But, modified how? In the present work, we investigate whether an ontology derived from quantum field theory can help resolve the hard problem. We begin with the assumption that experience cannot exist without being accompanied by a subject of experience (SoE). While people well versed in Indian philosophy will not find that statement problematic, it is still controversial in the analytic tradition. Luckily for us, Strawson has elaborately defended the notion of a thin subject-an SoE which exhibits a phenomenal unity with different types of content (sensations, thoughts etc.) occurring during its temporal existence. Next, following Stoljar, we invoke our ignorance of the true physical as the reason for the explanatory gap between present day physical processes (events, properties) and experience. We are therefore permitted to conceive of thin subjects as related to the physical via a new, yet to be elaborated relation. While this is difficult to conceive under most varieties of classical physics, we argue that this may not be the case under certain quantum field theory ontologies. We suggest that the relation binding an SoE to the physical is akin to the relation between a particle and (quantum) field. In quantum field theory, a particle is conceived as a coherent excitation of a field. Under the right set of circumstances, a particle coalesces out of a field and dissipates. We suggest that an SoE can be conceived as akin to a particle-a SelfOn—which coalesces out of physical fields, persists for a brief period of time and then dissipates in a manner similar to the phenomenology of a thin subject. Experiences are physical properties of selfons with the constraint (specified by a similarity metric) that selfons belonging to the same natural kind will have similar experiences. While it is odd at first glance to conceive of subjects of experience as akin to particles, the spatial and temporal unity exhibited by particles as opposed to fields and the expectation that selfons are new kinds of particles, paves the way for cementing this notion. Next, we detail the various no-go theorems in most versions of quantum field theory and discuss their impact on the existence of selfons. Finally, we argue that the time is ripe for a rejuvenated Indian philosophy to begin tackling the three-way relationship between SoEs (which may become equivalent to jivas in certain Indian frameworks), phenomenal content and the physical world. With analytic philosophy still struggling to come to terms with the complex worlds of quantum field theory and with the relative inexperience of the western world in arguing the jiva-world relation, there is a clear and present opportunity for Indian philosophy to make a worldcentric contribution to the hard problem of experience.
\end{abstract}




\section{The hard problem of consciousness}

In its simplest formulation, the hard problem of consciousness is stated as "Why is anything accompanied by experience?". To elaborate using naturalistic language, why are some physical processes and events associated with an interior-a qualitative space which is felt from the inside. The presence of experience forces us to acknowledge that nature has an interior, private and qualitative space and that there is no obvious a priori reason for this to exist. In recent times Chalmers [5] often gets the credit, and rightly so, for putting the hard problem in its starkest terms. The hard problem of consciousness does not imply a Cartesian subject or soul as the rest of this paper should make clear. Further, and this is even more relevant to an Indian audience, no a priori transcendental idealist stance can dissolve the hard problem: the key issue is interiority pure and simple and this needs to be explained. For a physicalist the problem is "why are physical processes accompanied by experience?" and likewise for a transcendental idealist, the problem is "why is Brahman accompanied by interiority?", though the latter is not something that one hears very often.

The physicalist response of the past few decades has mainly been emergence [9]. My colleagues for example, constantly seek to reassure themselves by asserting that under the right set of conditions and at the appropriate level of complexity, consciousness pops out from brain activity. The conditions are rarely spelled out: "We don't know enough about the brain" is the refrain and the complexity measures (and necessary thresholds) never formulated. Two other defense mechanisms stand out. If it is pointed out that brain mechanisms are actually supervenient on a more basic level of physics, the charge is one of reductionism. If new relations are postulated to bridge the explanatory gap between brain processes and experience, the charge is one of sui generis interactionism. With these two moves, further philosophy is discouraged and tacit commitments to naïve identity theories left unchallenged and in their place-with the complexity problem of emergence left unaddressed. Henceforth, we use the term taboo physicalism for this approach cluster since it encompasses emergentist and eliminativist approaches alike while seeking to corral the mind-body problem for its exclusive preserve. Emergence (and eliminativism [7]) should be carefully separated from holism (as will become clear). The various emergentist approaches usually get started by pointing out the existence of lively self-organizing phenomena which adhere to the second law of thermodynamics in letter while violating its spirit. The mind can then be seen as "popping out" from the brain at a suitable level of complexity. In sharp contrast, holism asserts that compositionality is not mere combination and that mind could be a holistic property of matter. Holism goes back to Aristotle in the west and seems to be popular in Indian circles but it is fair to say that mind-body holism has not seen a full fledged defense in recent years. For these reasons, we separate holistic physicalism from taboo physicalism introduced earlier.

Idealism has seen better days in western philosophy. With roots stretching back several hundred years [30], one would have been led to expect a strong response from the idealist camp by taking a cue from Bishop Berkeley and adding German and Indian idealism to the mix. And, arguably there has not been a strong response from Eastern (and Indian) idealism either. As we have argued above, the onus is on the transcendental idealist to explain why there "seems" to be spatio-temporally bounded interiority (experience) in the universe or in lila. Further, and this is again just based on anecdotal observation, recently it has become somewhat fashionable to assert that the universe is a simulation and this grounds idealist intuition. First, without additional underpinnings, the simulated reality hypothesis is still physicalist (with a quantum computer [21] or equivalent generating the simulation). Second, the question of why such a simulation is accompanied by experience still needs explanation. In other words, the explanatory gap persists in this setting as well despite the virtual reality twist. 
In recent years, a surprisingly strong response has came from panpsychism [29, 33, 30]-the ancient doctrine that qualia extend all the way down in nature and savaged by the critique that this meant that "rocks have feelings." In hindsight, this robust response is not surprising. Since complexity thresholds for the emergence of experience are hard (if not impossible) to come by, the alternative that consciousness gradually fades as one goes down in scale was always viable. In other words, interiors are complex and gradually become more and more simple as one goes to smaller scales. Complexity per se does not lead to consciousness. Instead consciousness gradually becomes more complex on an evolutionary time scale. Panpsychism faces the combination problem [26]: how do proto-experiences combine to form the full fledged experiences of which we are familiar. Or to put it in a different way: more complex experiences do not seem to be built up from simpler experiences in the same way that, say, polypeptide chains are made up of atoms. This is an active area in philosophical circles at present. Panpsychism has to explain why consciousness is spatio-temporally localized and is a very rare occurrence in nature-unless our intuitions in this matter are seriously misguided. It may turn out that a sophisticated panpsychism will eventually emerge which is neither pan nor "psychic" but ends up assigning proto-experientiality to a small subset of nature. To summarize, while panpsychism is a genuine alternative to emergence, it faces huge problems of its own. This response to emergence also begs the question: why can't we begin with a revamped physicalism which is not emergentist and is not a priori panpsychist? Our efforts here belong in this camp.

Another sophisticated response is neutral monism [2] stated simply as "Mind and matter are manifestations of something neutral." Here, the challenge is the explication of neutral properties, substances etc. and how they give rise to both mind and matter. Further, there's an asymmetry between mind and matter which is not addressed in neutral monism. There is far more matter than mind in the universe. And, it's not clear why we can't begin with a physicalism which eschews matter as a starting point while accommodating experience. It is exactly the lack of a deeper physicalist theory which is the problem in most versions of neutral monism. Due to this, neutral monism often slides into a dual-aspect theory [1] wherein the interior aspect of nature is pushed all the way down with interior/exterior parallels established at various levels. Such a pan-interiority clearly has echoes in panpsychism which is why neutral monism, dual aspect theory and panpsychism are often discussed together. Clearly there are numerous differences between these myriad approaches. Since the present work's focus is an expanded physicalism [31] that can accommodate experience, we choose not to focus on the doctrinal differences in the above and instead move on to presenting our case.

Panpsychism's strength is that it takes consciousness as fundamental. Neutral monism goes beyond both matter and mind in search of something more fundamental. Dual-aspect theory considers nature's interiority sacrosanct. Panpsychism faces the combination problem: how do experiences combine. Neutral monism faces the fundamental problem of a lack of a deeper theory to ground the neutral "substance." Dual-aspect theory faces the problem of an a priori split between interior and exterior which is left unexplained. Finally, emergentist approaches still remain true to their anti-reductionist origins while facing the complexity problem. The expanded physicalism we envisage here has much in common with all four. The goal of this work is to suggest an expansion of physicalism [32] which, while accommodating experience, remains plausible. Indeed, we invoke ignorance of the true physical in embarking on this course. It is here that quantum field theory (QFT) ontologies are especially useful. Since the ultimate constituents of nature are still a mystery in QFT, we face the unusual situation of a physical theory which is effective empirically but whose physicalist underpinnings remain murky. In what follows, we examine QFT ontologies in search of ways in which experience can be accommodated. Before this however, we need to first shore up the basic phenomenological intuition informing our physicalist expansion. 


\section{Subjects of experience}

If the intuition behind the hard problem is unclear to the reader, we suggest the following experiment. Pinch yourself....hard, but not so hard as to hurt yourself (unless you're a masochist and are into that sort of thing).

Done? As the (hopefully mild) pain recedes into the horizon, notice the immediacy, interiority and qualitative feel (qualia) of the pain-which has an onset, a peak and decay. It is also spatiotemporally localized. When you pinch yourself, other people don't feel the pain (or don't appear to). For these reasons, the first phenomenological intuition guiding our approach is that experience is always associated with a subject of experience (SoE). This is hugely controversial in analytic philosophy but often taken for granted in Indian philosophy. In the Anglo-American tradition, the harsh dualism associated with Descartes of (eternal) soul and (temporal) body has largely made talk of SoEs unpalatable. Recently, however, Strawson [34] has made a yeoman effort in resurrecting the more limited concept of a "thin subject." A thin subject is an SoE which is spatially and temporally bounded, arises and decays and has phenomenal content associated with it during its (typically) short existence. From an eastern perspective, a thin subject is a (Mahayanist?) middle ground between the extremes of an eternal subject (often seen in Vedanta) or selfless experiential dharmas (often seen in Theravada). Rather than try and correlate the thin subject with the mere self (and other nominal entities) [15], we reiterate Strawson's point that the thin subject cannot be easily denied. A thin subject of experience-exactly THAT which is conscious of reading this sentence right NOW-is a center of awareness, necessarily separate from the phenomenal content which arises in awareness [6]. Strawson takes this as a given and enshrines it as a thin subject of experience. The natural concern here is the doubling of mysteries: we don't understand experience and adding an SoE to the mix seems to unnecessarily complicate matters. But, following Strawson, our strategy will be to draw an analogy between the SoE/phenomenal content/experience cluster and the object/property/process cluster. Insofar as we face serious problems with the concepts of objects, properties and processes, we can expect to face the same in the consciousness cluster. The difference is the apparent lack of relationship between SoEs and physicalism. If an SoE can be seen as a certain type of object in an expanded physicalism, then an experience can be unpacked as a spatio-temporal property of this new type of object. To prefigure things, we plan to ground SoEs by suggesting that QFT ontologies can support a new relation in which an SoE can be seen as akin to a new kind of particle. Since QFT ontologies are still in flux, this does not entail a commitment to a new substance nor does it imply that the new particles are mere properties.

Subjects of experience do figure in western neo-Aristotelian traditions (as opposed to the analytic tradition). Recently, Lowe [22] and van Inwagen [36] have attempted to take into account SoEs in a neo-Aristotelian metaphysics. van Inwagen, in particular, poses the special composition question which we present (in modified form) below: can an SoE be seen as a new whole but composed of existing parts such that the new whole has phenomenal properties associated with it? The difficulty with this question is that it is not clear what we mean by "existing parts" since the physicalist base may itself be unknown. The second difficulty is that "the whole is greater than the sum of the parts" implies a new conception of compositionality or holism which must be adequately defended before this project can get off the ground. Recall that we mentioned earlier the difference between holistic and taboo physicalism. van Inwagen, in posing the special composition question, is clearly hoping for a holistic theory (if not a physicalism) which we have reconfigured to try and address the problem of experience. Such an approach raises compositionality up as a new principle of wholeness (with emergence being its natural contrast). Or as a slogan, experience is 
a holistic and not an emergent property of the brain. The question for us then is this: can a new compositionality relation connect SoEs to the physical? And, how is this different from being yet another emergent property?

To answer this question, we turn to the recent work of Koslicki [16]. More than any other person, Koslicki, in recent times, has rescued compositionality from becoming irrelevant. Lack of space does not permit us to do full justice to her work, so we attempt a brief summary. Koslicki has shown that a new object can be distinguished from its parts provided there are independent reasons for believing that the new object exists in the first place. For Koslicki, if the new object can be shown to be a member of a natural kind [20], then a weak supplementation principle can be invoked to relate the object to a set of material and formal parts. (The presence of formal parts in the new object clearly marks this approach as a neo-Aristotelian one.) In an exchange, rare in analytic philosophy, Koslicki engaged in a debate with an opponent who held the view that arbitrary wholes could be formed by part combination (thereby rendering the concept toothless). The upshot was that if objects can first be justified on natural kind grounds, then weak supplementation could be invoked as described above. This raises the question: what is a natural kind as opposed to an artifactual kind and what do natural kind terms pick out in this world? Unfortunately, this is a very deep subject with the arguments mostly driven by modal logic. Instead we merely note that natural kind terms are very prevalent in biology ("a neon tetra is a kind of fish") and we are seeking here to apply the same common sense approach to the problem of experience (and therefore ultimately to psychology). We note the presence of the individual and the collective in the fleshing out of a natural kind. Finally, it should be clear that biological natural kind objects are clearly composites but so are motorcycles (Koslicki's example). Since we are only concerned with natural kind objects, there will be no need for discussion of the holistic properties (of the lack thereof) of artifactual objects like motorcycles. The former are our concern and not the latter since we seek to connect SoEs to natural kind objects.

We have independent reasons for believing that SoEs are natural kind objects in the (physical) world. Even the most extreme solipsist would probably (inadvertently and occasionally) grant subjecthood to another person in dialog. And, due to the fundamental role played by second person intersubjectivity in human culture [13], the notion that SoEs form a natural kind should not be too controversial (provided one buys into the SoE concept in the first place). For at least two decades, the subdivision of consciousness studies into first, second and third person has been in place. Consequently, the notion that the second person "experience" acts as a bridge linking different first person experiences can be taken for granted. Note that we are not attempting to explain intersubjectivity here. Instead, we again appeal to common sense and note that we are aware of "the other" via the second person experience. Despite our inability to directly see into the interior of another person, we accept the existence of the other with experiences similar to ours. If this is accepted, then we have, per Koslicki, independent grounds for connecting SoEs to the physical via a new compositionality relation. The work is not yet done since the specifics of the new relation are where the real payoff will occur. And it may turn out that the new relation is not explicitly a neo-Aristotelian compositionality relation. All we really require is the grounding of SoEs as new natural kind objects which have to be related to the physical. We need to carefully expand physicalism in such a way that SoEs (which are new natural kind objects) can be accommodated [25]. And since experience is always associated with an SoE, phenomenal content can be conceptualized as spatio-temporal properties of the new SoE objects. Regardless of the ultimate status of the object/property/process cluster, SoE objects have phenomenal properties and are part of a natural process. While this expansion of physicalism could have been denied had physicalism been complete, our ignorance of the true physical combined with the murky underpinnings of QFT allow for an expansion. 


\section{Quantum field theory ontologies and SelfOns}

Interpretations of quantum mechanics have been a mainstay in consciousness studies for at least thirty years [38]. The lack of a single interpretation acceptable to nearly everyone has played a role in stoking the flames. Quantum mechanics has strange features: (i) a fundamental uncertainty, (ii) wave function collapse, (iii) possibilities rather than trajectories and (iv) entanglement, to name a few. Quantum mechanisms in the brain [12] have been suggested as playing a role in consciousness despite objections ranging from irrelevance to decoherence [10]. At this juncture, the orienting generalization for us is the simple fact that quantum mechanics (as opposed to quantum field theory) is a story regarding particles and how they (occasionally) behave like waves. We are interested in going in the opposite direction: how and when do waves (or more technically fields) behave like particles? The reason: in a field theory, particles cannot be seen as basic but instead as related to their fields. First quantization takes particles for granted and is focused on how they behave like waves in certain contexts like the two slit experiment or exhibit non-local correlation as in the EPR experiment. First quantization is still a mystery, undoubtedly due to Schrödinger's unusual change of variables approach [27] in a particle Lagrangian variational principle. Second quantization attempts to obtain particles from field Lagrangians and is the basis of quantum field theory. It is much less of a mystery with the basic recipe taught in QFT textbooks. In our approach, we seek to leverage our ignorance of the true physical in order to accommodate SoEs, thereby explaining experience. The essential new idea: in a nutshell, we would like to expand physicalism by conceiving of SoEs as akin to new kinds of particles related to a set of basic quantum fields.

The ontology of quantum fields is far from settled $[18,17]$. A simple reason is the presence of multiple QFTs: (i) standard QFT in Hilbert and Fock spaces, (ii) algebraic QFT and (iii) Feynman path integrals. Consequently, it is difficult to firm up metaphysical commitments. However, regardless of the availability of competing QFTs, an orienting generalization we can make is that any QFT ontology will have to deal with fields, particles and their relations. QFT (other than Einstein's theory of gravity) is one of the most empirically successful physical theories. Indeed the standard model of particle physics [28] (which does not include gravitation) is one of the most rigorously tested QFTs. The division of matter into fermions (quarks and leptons) and bosons (Higgs, W and Z, photons and gluons), their relations to fermionic and bosonic fields and their inter-relationships are taught in standard textbooks. Particle interactions in the presence of three forces (strong, weak and electromagnetic) are mediated by corresponding bosons (gluon, W \& Z and photon respectively). During the course of cosmic evolution, the quantum gravity epoch (where gravity and the other forces are unified) gives way to the grand unification epoch (where the three forces are unified but separate from gravity). This in turn leads to the inflationary epoch (in which the strong force separates from the electro-weak) and then the quark epoch (where the three forces in the standard model have all separated and are distinct from gravity). The sequence of symmetry breakings which cause a unified field (except for gravity) to separate into three fields and their associated bosons and fermions is well understood. The particle-field relation plays a fundamental role in all epochs with forces between fundamental fermions mediated by bosons. Indeed, a standard move in contemporary textbooks is to present second quantization as a way in which quantum fields behave like particles. In this view, all particles arise out coherent field excitations, persist and decay (except for the photon).

The particle-field relation is central to QFT. Fundamental issues remain as to whether particles and fields are substances, tropes, properties or something else entirely. There are important nogo theorems in QFT such as Malament's $[23,17]$ which seem to exclude the possibility of spatiotemporally localized particles at all. These are typically augmented with FAPP (for all practical purposes) principles in which particles reappear with a FAPP "as if" status despite the underlying 
theory not allowing for their possibility. Some authors have been driven to argue that particles in QFT are supervenient on quantum fields and their appearance in empirical measurements have merely a FAPP status. A second concern regarding fundamental particles is indistinguishability with Wheeler quipping that there was only one electron in the universe. To the best of our knowledge, QFT ontologies have not progressed to the point of posing and answering the question of the existence of sharp, spatio-temporally localized, distinguishable particles. The intriguing aspect of QFT ontologies from our perspective is the ability of the universe to manifest particles from field excitations as opposed to particles being the sole foundation in nature. Whether individual particles are spatio-temporally localizable or not is secondary; likewise for indistinguishability.

We have now arrived at the point of payoff. We wish to appeal to QFT ontologies in support of setting up a new relation connecting SoEs to the physical. In this new conception, SoEs are akin to new particles-SelfOns - that arise out of basic fields, persist and dissipate. Selfons mirror the phenomenology of a thin subject and are associated with phenomenal content (thoughts, feelings) which are now conceived as new spatio-temporal properties. Selfons can also be seen as objects of a new natural kind which are always accompanied by experience. Fundamental issues of indistinguishability, spatio-temporal localization, sharpness and the viability of the object/property/process cluster will all have to be worked out within the framework of a QFT ontology: likewise with the issue of whether selfons are supervenient on a more basic QFT ontology. We seem to have answered our question in the affirmative: there does not appear to be (at present) a knockdown argument against conceiving of SoEs as selfons. The ignorance hypothesis (of the true physical) permits the construction of a plausible new physicalism which includes selfons. As long as we have independent evidence of SoEs (from phenomenology and intersubjectivity) followed by the conception of SoEs as objects of a new natural kind-selfons-there does not appear to be any barrier to their inclusion into physicalism. Clearly, this is a scientific hypothesis driven by a philosophical starting point (physicalism) and therefore could be wrong. For example, while we have asserted that selfons are akin to new particles, perhaps the QFT-selfon relation is totally different. We feel that it is important to begin with the above selfon hypothesis at this juncture for the reasons of (i) simplicity and (ii) grounding in present day QFT.

All the standard objections apply: here's a sample. (i) Basic physics is settled with no place for selfons; (ii) This is just neutral monism with fields being the neutral entity and ordinary particles and selfons corresponding to matter and mind respectively; (iii) There are no sharply localized particles, hence no selfons in principle; (iv) You have gotten confused between particles and states and your approach actually suggests that consciousness is a new state of matter; $(v)$ This is reductionist to the core since you have reduced consciousness to (new) particles. There is no space to address all these (good) objections. Instead, we settle for a brief rejoinder to (i) above. We take it as a given that basic physics is not settled and that (despite string theory), we are still far away from the grand unification of gravity with QFT. When we describe selfons as akin to particles, we should clarify that these new "particles" could better be conceived as composites rather than fundamental. But, composites how? Note that we alluded to a weak supplementation principle of holism pioneered by Koslicki. Selfons could arise from a new holistic principle linking basic fields to new particles (as opposed to being compounds made up of existing fermions and bosons). This is one way things could go. But this way has already been pointed out by Lowe, van Inwagen, Strawson and Koslicki. All that remains is for physicalism to follow this lead. While this is certainly a very difficult task, it is not in principle impossible. This route also bypasses historical divisions between matter and mind. We reiterate that the conception of SoEs as selfons is suggestive of a new organization principle linking basic fields to new particles (without being an emergent property of matter). Finally, this is actually an attempt to do both philosophy and physics with the end result open to multiple interpretations-due to the unsettled nature of QFT ontologies. 


\section{A criticism of and an opportunity for Indian philosophy}

We close by pointing out the relevance to Indian philosophy since that setting is presumably more familiar to the audience than continental or Anglo-American philosophy. A sharp criticism is first offered which will hopefully be seen as tough love rather than condemnation. This is followed by a discussion of the new opportunities presented by the hard problem of consciousness in general and QFT ontologies in particular.

\subsection{Criticism}

Given the fundamental (and some would say insurmountable) difficulty posed by the hard problem, one would be led to expect an appropriate response from Indian philosophy especially when the literature's obsession with the Atman-Brahman relation for over two and a half millennia is taken into account. This is not to be found. Dialog with Indian philosophers (and this is clearly anecdotal) reveals the usual mystical and idealist leanings and a failure to take the hard problem seriously. As we have previously mentioned, even when we restrict focus to transcendental idealism, there is no sustained exploration or rapprochement with the idealisms of Berkeley, Fichte, Schopenhauer [4] etc. When one repeatedly hears the statement that consciousness is fundamental, the world is an illusion and therefore there is no hard problem, it's clear that there is no appreciation of the limited, localized, interior, private and ineffable nature of qualia and the need to relate them to something beyond. If the reader at this point continues to think that some form of absolute idealism resolves the hard problem, please note that the split between subjective, spatio-temporally bounded qualia and the world still needs to be explained even in an idealist metaphysics. And, despite the Indian literature's obsession with awareness (as opposed to phenomenal content), we have not seen any exploration of the triadic relationships between SoEs, phenomenal content and the world. As we have mentioned above, recently there has been an uptick of interest in treating the universe as a simulation. Regardless of the physical basis of the simulation, in this picture, there is a clear and pressing need to relate virtuality and consciousness [14]. We have not seen much exploration in Indian philosophy of treating the world as virtual (and not merely as an illusion) and attempting to situate subjective experiences within such a world. Philosophers trained in the analytic tradition continue to express their frustration with Indian philosophy in private. The criticisms range from: (i) it is a cul de sac with no interest in other traditions; (ii) it has become dominated by different schools of Vedanta [8] leaving Nyaya, the Carvaka materialists and the Samkhya dualists [35] behind; (iii) despite the rise and rise of worldcentric Buddhism in the past forty years, there's no adequate response from Indian philosophy; (iv) no attempt has been made to connect the recent interest in panpsychism with primordial Indian philosophical traditions; (v) too much material remains trapped in Sanskrit with no interest shown in translation and dissemination; (vi) Indian philosophers (and to be clear, this criticism is mainly aimed at Indian philosophers disseminating Indian philosophical views) have a tendency to keep repeating their talking points and remain unable to engage with a radically different audience; (vii) the (usually tacit) idealist metaphysics hobbles and curtails all discussion to the jiva-Brahman relation and does not permit exploration of the jiva-world relation.

\subsection{Drawing inspiration from Buddhism}

We attempt to elaborate on (iii) above in an impressionistic manner. Prior to the ' 80 s, there was not much engagement between Buddhism and the west. The explosion of interest in new paradigm (mainly non-reductionist) thinking and the rise of postmodernism in the '80s allowed Buddhism to establish a beachhead in the western academy [24]. Facile analogies between the Buddhist 
(Abhidharma) no-self doctrine and emergentist (and embodied) theories of consciousness [37, 39] further led to the dissemination of Buddhist ideas. The same was not true of Vedanta and Indian philosophy in general (but with some exceptions [11]). The comparatively more theistic leanings of Vedanta coupled with the culture wars in the US and Canada ensured that American Vedanta, for example, did not have as large a footprint as American Buddhism. Consequently, while Buddhism became the de facto standard bearer (for Eastern philosophy) in consciousness conferences, Vedantic and other Indian philosophical concepts did not have a similar impact. Buddhism has faced serious problems in its transition from specific Eastern traditions to becoming a worldcentric religion and philosophy. Take, for example, the embodied self arguments in [37]. After first deconstructing the self in standard Buddhist fashion using Abhidharma concepts, the authors back away from these arguments by deploying the Madhyamika (shunyata) no-view dialectic on the Abhidharma. In other words, the sharp and clear no-self, Abhidharma doctrine gives way to an emptiness of all views, Madhyamika approach. Despite the danger of using emptiness as a "crowbar" to justify a certain view, the authors return at the end of the book to embodiment of mind as their position. This allow the authors to (a) introduce Buddhist concepts like shunyata to a wider and secular audience while (b) promulgating a particular view-embodiment. In the two plus decades since the book's publication, Abhidharma and shunyata have entered the consciousness lexicon but other Buddhist doctrines that build on these approaches have not. It is quite common to encounter people at consciousness conferences discussing Buddhist no-self doctrines and arguing that the emptiness doctrine should properly be viewed as mysticism but one rarely hears discussion of Buddhist tantra or Chinese Hua-Yen [3] for example. Admittedly, these are impressionistic and anecdotal observations, but it should be possible to perform data analytics on this point for confirmation. The take away for Indian philosophy is to follow the example of Buddhism's transition from a diverse set of Eastern cultures to a more unified, worldcentric one.

\subsection{Opportunity}

If the above criticisms and impressions are on the mark, Indian philosophy has a huge opportunity. Despite a rich, vibrant and much better funded philosophical tradition, continental and AngloAmerican philosophy are in crisis. Their continued inability to create a satisfactory quantum field theory ontology is perhaps not an accident and may be due to a foundational mismatch between a two and a half millennia western philosophical tradition founded on atomism and modern (and postmodern) physics. With QFT underpinnings up for grabs, it is astonishing to see continued talk of the matter-mind split given the swampland foundations of matter. Too much of present day analytic philosophy still remains obsessed by interpretations of quantum mechanics instead of delving into QFT and formulating exactly what is meant by a particle. The arrival of the new century has seen the calls for "Everything must go" [19] at the foundations which are clearly indicative of something amiss. Indeed, just as Indian philosophy has been mired in mining the Atman-Brahman relation, the west has been bogged down by the matter-mind split. If as we have suggested, the matter-mind (or subject-object) split cannot be solved without shaking up the foundations of matter (via QFT ontologies and the like) and without re-introducing subjects of experience into the mix, then western philosophy may be ripe for a revolution. It is here that Indian philosophy can play a worldcentric role by (i) arguing the jiva-world relation using all of the tools at its disposal, (ii) setting up a three-way relationship between the world, jivas and Brahman and finally (iii) bringing back the other schools of Indian philosophy [35] who have been neglected due to the univocal emphasis on Vedantic idealism. (Western scholars do not seem to be aware of the qualified nondualism of Vishishtadvaita and the dualism of Dvaita for example and think that Advaita is the only game in town.) First, selfons offer a bridge between the jiva and the world. The phenomenology 
of a selfon includes awareness of phenomenal content (thoughts, feelings, visualizations, sensations etc.). Since selfons are objects of a natural kind, the hermeneutics (cultural and mutual co-creation) of selfons include intersubjectivity and proto-language. Next, the structure of a selfon is based on a new compositionality relation with fundamental fields (and is foundationally different from that of matter). In contrast to a cartoon Cartesianism, the temporal nature of a selfon is that of a thin subject which lasts for only a few seconds. As opposed to emergentist theories, selfons are constituted via a new (holistic) compositionality relation. A potential slide to neutral monism is avoided by asserting an asymmetry between mind and matter and by preserving a physicalist foundation. Dual-aspect theory is transcended as selfons can interact with the world (with both being physical). The approach is explicitly physicalist without being a priori panpsychist. The triad-selfons, phenomenal content and the physical world-serves as a bridge from physicalism to different schools of Indian philosophy whereas such a bridge was non-existent between materialism and Indian idealism. Finally, it is the present day ignorance and uncertainty regarding the foundations of QFT that allow us to expand physicalism in this manner. There may be unexpected resonances between QFT ontologies and Indian philosophy that are yet to be fleshed out. Issues such as indistinguishability and no-go theorems on sharp, localized particles may point to very intriguing connections between the selfons that form a natural kind and the nonlocal self in Indian traditions. For example, if there's no way to have a sharp, spatio-temporally localized, distinguishable particle in QFT, then this may apply to selfons as well. In that case, selfons would in principle be indistinguishable and we can follow Wheeler down the rabbit hole and assert that "there is only one SelfOn" despite the FAPP insistence on the existence of many. (We do not need to point out the relevance of the many actually being one to Indian philosophers.) While there's a tendency among many Indian scholars to assume that metaphysics is settled (undoubtedly due to the weight and age of the tradition), the unsettled nature of QFT ontologies should give them pause. Finally, given the historical impact Indian idealism has had on European philosophy, a revamped Indian materialism (with aid from the other Indian philosophical traditions) may be well positioned to make worldcentric contributions to the hard problem of consciousness. 


\section{References}

[1] H. Atmanspacher. Dual-aspect monism à la Pauli and Jung. Journal of Consciousness Studies, 19(9-10):96-120, 2012.

[2] E. C. Banks. The realistic empiricism of Mach, James, and Russell: Neutral monism reconceived. Cambridge University Press (Reprint edition), 2016.

[3] T. Cleary. Entry Into the Inconceivable: An Introduction to Hua-Yen Buddhism. University of Hawaii Press, 1983.

[4] S. Cross. Schopenhauer's encounter with Indian thought: Representation and will and their Indian parallels. Monographs of the Society for Asian and Comparative Philosophy. University of Hawaii Press, 2013.

[5] D. J. Chalmers. The conscious mind: In search of a fundamental theory. Philosophy of Mind. Oxford University Press, revised edition, 1997.

[6] A. Deikman. 'I'=awareness. Journal of Consciousness Studies, 3(4):350-356, 1996.

[7] D. C. Dennett. Consciousness explained. Back Bay Books, 1st edition, 1992.

[8] E. Deutsch. Advaita Vedanta: A philosophical reconstruction. Studies in the Buddhist Traditions. University of Hawaii Press, 1980.

[9] A. Freeman, editor. The emergence of consciousness, volume 8 of Journal of Consciousness Studies. Imprint Academic, UK, 2001.

[10] D. D. Georgiev. Falsification of Hameroff-Penrose Orch OR model of consciousness and novel avenues for development of quantum mind theory. NeuroQuantology, 5(1):145-174, 2007.

[11] D. R. Griffin and H. Smith. Primordial truth and postmodern theology. Suny Series in Constructive Postmodern Thought. New York University Press, 1990.

[12] S. R. Hameroff. Quantum computation in microtubules? The Penrose-Hameroff "Orch OR" model of consciousness. Philosophical Transactions of the Royal Society A, 356:1869-1896, 1998.

[13] S. Hargens. Intersubjective musings: A response to Christian de Quincey's 'The promise of integralism'. Journal of Consciousness Studies, 8(12):35-78, 2001.

[14] D. Hoffman. Conscious realism and the mind-body problem. Mind \& Matter, 6(1):87-121, 2008.

[15] T. Jinpa. Self, reality and reason in Tibetan philosophy: Tsongkhapa's quest for the middle way. Routledge Critical Studies in Buddhism. Routledge, 1st edition, 2002.

[16] K. Koslicki. The structure of objects. Oxford University Press, reprint edition, 2010.

[17] M. Kuhlmann. The ultimate constituents of the material world: In search of an ontology for fundamental physics. Philosophische Analyse / Philosophical Analysis (Book 37). De Gruyter, 2010. 
[18] M. Kuhlmann, H. Lyre, and A. Wayne, editors. Ontological aspects of quantum field theory. World Scientific, 2002.

[19] J. Ladyman and D. Ross. Every thing must go: Metaphysics naturalized. Oxford University Press, 2009.

[20] J. LaPorte. Natural kinds and conceptual change. Cambridge Studies in Philosophy and Biology. Cambridge University Press, 1st edition, 2009.

[21] S. Lloyd. Programming the universe: A quantum computer scientist takes on the cosmos. Vintage, reprint edition, 2007.

[22] E. J. Lowe. Subjects of experience. Cambridge Studies in Philosophy. Cambridge University Press, 2006.

[23] D. Malament. In defense of dogma: Why there cannot be a relativistic quantum mechanics of (localizable) particles. In R. Clifton, editor, Perspectives on Quantum Reality: Non-Relativistic, Relativistic, and Field-Theoretic, volume 57 of Western Ontario Series in Philosophy of Science, chapter 1, pages 1-10. Springer Science and Business Media, 1996.

[24] Nāgārjuna. The fundamental wisdom of the middle way: Nāgārjuna's Mūlamadhyamakakārikā. Oxford University Press, 1995. Translated and Commentary by J. L. Garfield.

[25] A. Rangarajan. Panpsychism and compositionality: A solution to the hard problem. In The Science of Consciousness, 2016.

[26] G. Rosenberg. A place for consciousness: Probing the deep structure of the natural world. Philosophy of Mind. Oxford University Press, 2004.

[27] E. Schrödinger. Quantisierung als eigenwertproblem. Annalen der Physik, 384(4):361-376, 1926.

[28] M. D. Schwartz. Quantum field theory and the standard model. Cambridge University Press, 2013.

[29] D. Skrbina. Panpsychism in the west. MIT Press, 2007.

[30] T. L. S. Sprigge. The vindication of absolute idealism. Edinburgh University Press, 1st edition, 1984.

[31] D. Stoljar. Ignorance and imagination: The epistemic origin of the problem of consciousness. Philosophy of Mind. Oxford University Press, 2009.

[32] D. Stoljar. Physicalism, volume 2 of New Problems of Philosophy. Routledge, 2010.

[33] G. Strawson. Consciousness and its place in nature: Does physicalism entail panpsychism? Imprint Academic, 2006.

[34] G. Strawson. Selves: An essay in revisionary metaphysics. Oxford University Press, revised edition, 2011.

[35] P. R. Tigunait. Seven systems of Indian philosophy. Himalayan Institute Press, 1983.

[36] P. van Inwagen. Material beings. Cornell University Press, 1995. 
[37] F. Varela, E. Thompson, and E. Rosch. The embodied mind: Cognitive science and human experience. The MIT Press, revised edition, 1992.

[38] J. A. Wheeler and W. H. Zurek, editors. Quantum theory and measurement. Princeton Legacy Library. Princeton University Press, 1983.

[39] K. Wilber. Sex, ecology, spirituality: The spirit of evolution. Shambhala, Boston, MA, 1st edition, 1995. (page 708, note for page 497, chapter 14). 